\title{
Astronomy and Astrophysics Division
}

\section{- Inaugural Meeting}

\section{Rees, Cambridge, England}

The inaugural meeting of the Astronomy and Astrophysics Division of the European Physical Society was held at Meudon, France on 3-5 February 1972 , and was attended by over 150 scientists from twelve countries. The occasion also marked the official opening of the new Department of Fundamental Astrophysics at the Meudon Observatory. In addition to contributed papers, invited review papers were presented.

One session was devoted to radio astronomy. Van der Laan (Leiden) reviewed some studies of extragalactic objects made with the Westerbork array, which came into operation in 1970. Because of the high sensitivity of this telescope, maps of extended sources can be made so that radio contours can be determined down to lower surface brightness than with previous maps. These maps reveal that many double sources are joined to their parent object by faint 'bridges', which often display much higher polarization than the rest of the source. Some oddly-shaped sources in clusters of galaxies (3C465, for example) were interpreted by Van der Laan as 'galactic wakes' from continuous or repeated ejection of radio-emitting plasma by galaxies moving at $>1000 \mathrm{~km} \mathrm{~s}^{-1}$ through intergalactic gas. Some radio emission has been detected from all Shapley-Ames galaxies brighter than the tenth magnitude. Radio maps of nearby spiral galaxies (M51 and NGC4268, for example) have yielded important new insights into the origin of spiral structure. The evidence lends support to the 'density wave' theory developed by C.L. Lin (MIT) and his associates. Moreover observations of NGC4268 suggest how the formation of spiral arms might be initiated. It appears that two oppositely-directed jets of gas in the plane of the galaxy have been ejected from the nucleus. As these jets move outward, they sweep up gas from the spinning disc to cause a density en- hancement along a spiral track. This interpretation is based on continuous observations at $1400 \mathrm{MHz}$, and it will be tested when $21 \mathrm{~cm}$ line studies (which are planned for the near future) map the velocity distribution of the gas in NGC4268.

Mezger (Bonn) reviewed recent radio studies of interstellar gas in our galaxy. The gas is denser and cooler in spiral arms, and so star formation will be concentrated in these regions. Mezger also discussed the evidence from both hydrogen and molecular line observations in favour of an expanding ring of gas near the galactic centre.

Icna (Meudon) reviewed some aspects of infrared astronomy and described current plans for European work in this field. Pounds (Leicester) and Cavaliere (Frascati) reported on recent developments in $\mathrm{X}$-ray astronomy. Wolfendale (Durham) discussed the evidence from studies of extensive air showers concerning the spectrum and composition of cosmic rays with energies $>\sim 10^{15} \mathrm{eV}$. Computations reported by Osborne (Durham) render a galactic origin for these particles rather unlikely.

On more theoretical topics, Kundt (Hamburg) discussed the feasibility and precision of various methods of testing gravitation theories within the solar system. Some problems of the final stages of stellar evolution and the formation of pulsars were discussed by Paczynski (Warsaw). If supernovae are triggered by explosive ignition of carbon, current calculations suggest that the star would be completely disrupted, leaving no remnant behind; if a neutron star is to be left behind, some process is needed for making the explosion less efficient. Paczynski suggested that, if the core were convective, cooling by the URCA process would be more effective than had previously been assumed. This would delay carbon ignition until the core had evolved to a denser and more tightly-bound configuration.

\section{Business meeting}

At a business meeting, held after the scientific sessions, draft statutes were considered, and there was an extensive discussion of how the Division should develop. Members agreed that its role should be to promote fruitful interaction between astronomers and physicists, and to improve communications among astronomers and astrophysicists (especially the younger ones) in different European countries. It was suggested that further general meetings should be held periodically along the lines of the inaugural general assembly, and also that specialized meetings should be arranged (especially those where the expertise of physicists might usefully be brought to bear on some astronomical topic). Three specialized meetings and one summerschool sponsored by the Division are planned to take place later in 1972.

Proposals were discussed to establish two Sections of the Division, dealing with solar astronomy and cosmic rays respectively. It was agreed that, while the Division should sponsor meetings on these subjects, it was perhaps premature to establish Sections on a formal basis at this stage. The problems of disseminating information, especially in fields on the borderline of physics and astronomy, was emphasized. In particular, several members emphasized the usefulness of such works as Kuchowicz's bibliographies of nuclear astrophysics.

It was suggested that, in view of the activities and scope envisaged for the Division's work, an appropriate name would be 'Astronomy and Astrophysics Division'. About twenty persons were nominated as candidates for the Board of the Division, which will replace the present Interim Steering Committee. An election is now in progress to choose twelve Board Members. 